\title{
Cooperativity and Site-Selectivity of Intramolecular Hydrogen Bonds on the Fluorescence Quenching of Modified GFP Chromophores
}

Deng-Hsiang Chang, ${ }^{\dagger}$ Chun-Lin Ou, ${ }^{\dagger}$ Hung-Yu Hsu, ${ }^{\dagger}$ Guan-Jhih Huang, ${ }^{\dagger}$ Cheng-Yi

Kao, ${ }^{\dagger}$ Yi-Hung Liu, ${ }^{\dagger}$ Shie-Ming Peng, ${ }^{\dagger}$ Eric Wei-Guang Diau, ${ }^{\dagger}$ and Jye-Shane Yang ${ }^{*}{ }^{\dagger}$

Department of Chemistry, National Taiwan University, Taipei 10617, Taiwan

Department of Applied Chemistry and Institute of Molecular Science, National Chiao Tung University, Hsinchu 30010, Taiwan

jsyang@ntu.edu.tw

(Supporting Information 18 pages)

Table of contents:

Normalized absorption and emission spectra of $\mathbf{1 0 H}-\mathbf{3 O H}$ and

$\begin{array}{ll}\text { 1OMe-3OMe in } \mathrm{CH}_{3} \mathrm{CN} & \mathrm{S} 2\end{array}$

Normalized absorption spectra of $\mathbf{1 0 H}-\mathbf{3 O H}$ in $\mathrm{MeOH} \quad \mathrm{S} 3$

Normalized fluorescence spectra of $10 \mathrm{H}-30 \mathrm{H}$ and $10 \mathrm{Me}-30 \mathrm{Me}$ in methylcyclohexane $\quad \mathrm{S} 4$

Crystallographic data of $\mathbf{1 0 H}, \mathbf{2 0 H}$ and $\mathbf{3 O H} \quad$ S5-6

Thermal ellipsoid plots for $\mathbf{1 0 H}, \mathbf{2 O H}$ and $\mathbf{3 O H}$ S7-9

Unit cell packing of $\mathbf{1 0 H}, \mathbf{2 O H}$ and $\mathbf{3 O H} \quad \mathrm{S} 10-12$

$\begin{array}{lr}{ }^{1} \mathrm{H} \text { and }{ }^{13} \mathrm{C} \text { NMR spectra of } 4 & \text { S13-14 }\end{array}$

$\begin{array}{ll}{ }^{1} \mathrm{H} \text { and }{ }^{13} \mathrm{C} \text { NMR spectra of 3OMe } & \text { S15-16 }\end{array}$

${ }^{1} \mathrm{H}$ and ${ }^{13} \mathrm{C}$ NMR spectra of $\mathbf{3 O H} \quad$ S17-18 


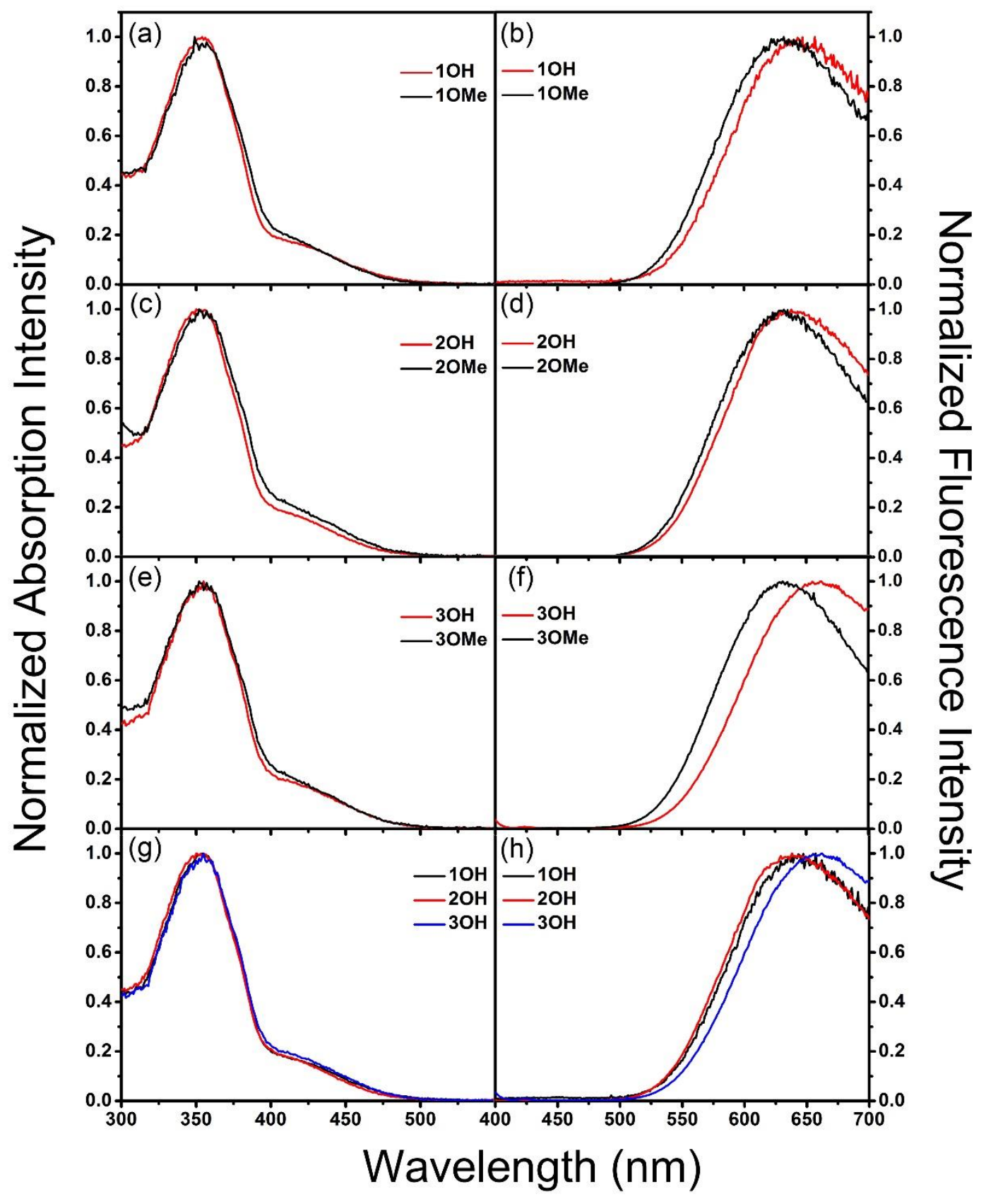

Figure S1. Normalized absorption and emission spectra of 10H-30H and 10Me30me in $\mathrm{CH}_{3} \mathrm{CN}$. 


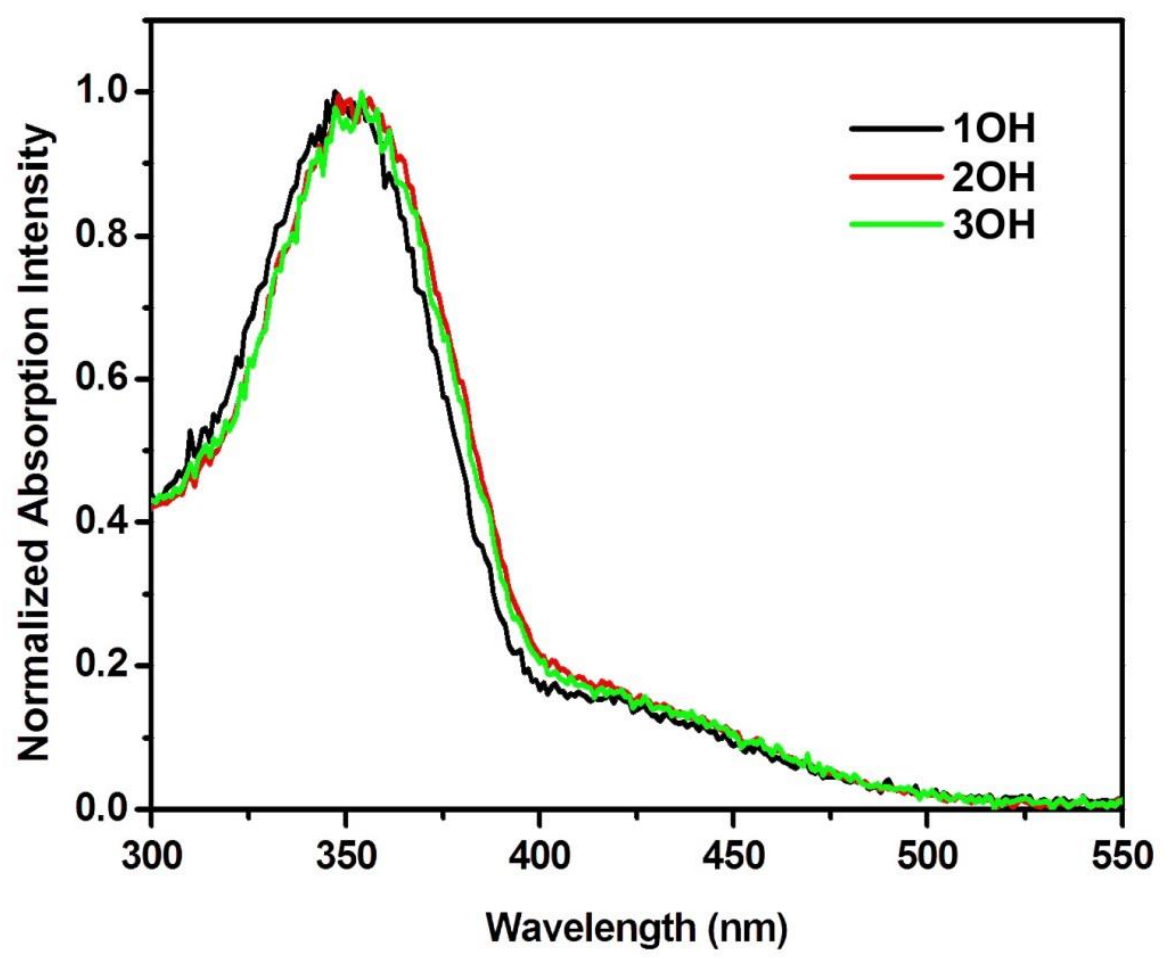

Figure S2. Normalized absorption spectra of $\mathbf{1 0 H}-\mathbf{3 O H}$ in $\mathrm{MeOH}$. 


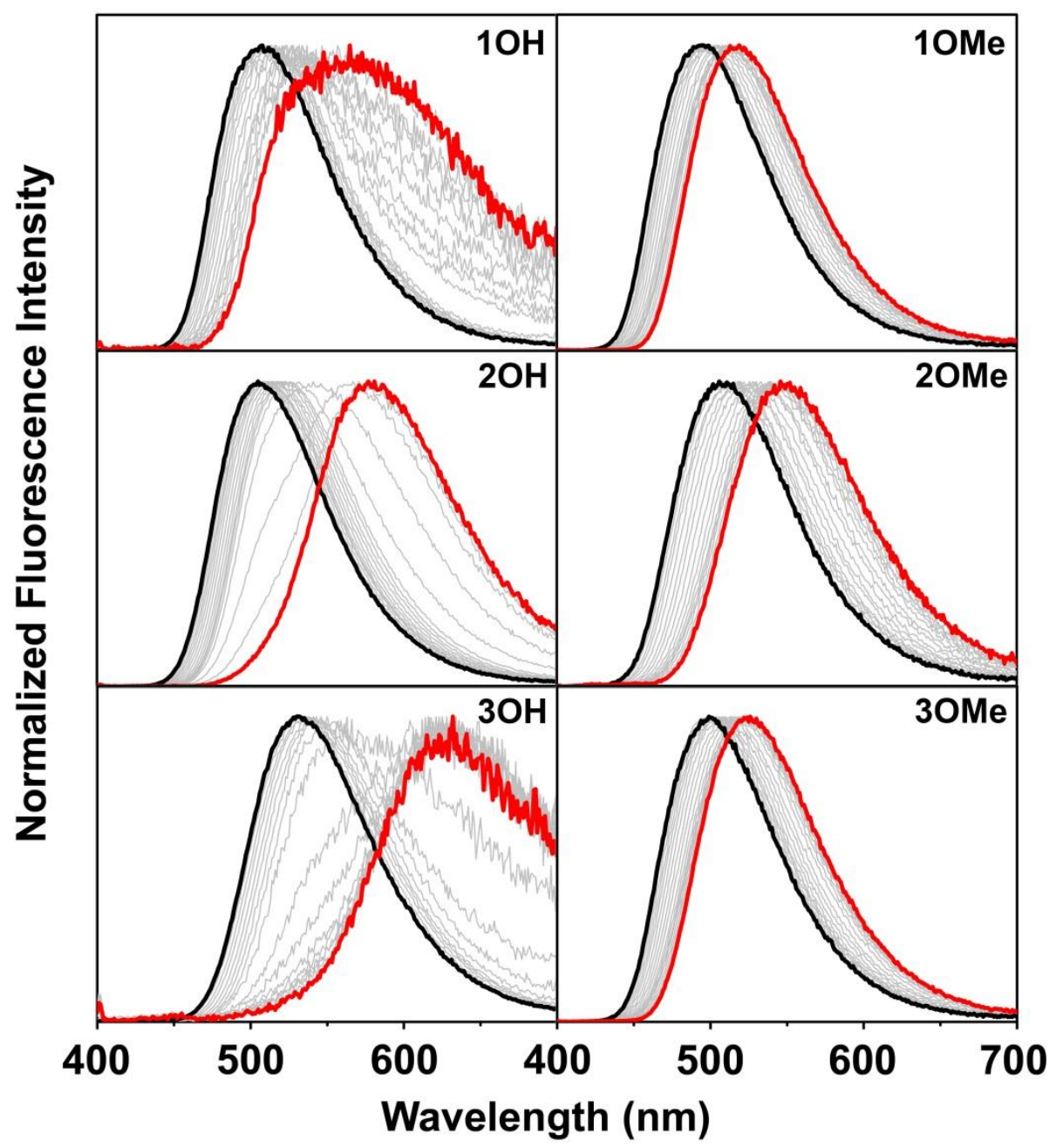

Figure S3. Normalized fluorescence spectra of $10 \mathrm{H}-30 \mathrm{H}$ and $10 \mathrm{Me}-30 \mathrm{Me}$ in methylcyclohexane. The spectra were recorded from $298 \mathrm{~K}$ (black) to $128 \mathrm{~K}$ (red). excitation wavelength is $350 \mathrm{~nm}$. 
Table S1. Crystallographic data of $\mathbf{1 0 H}-\mathbf{3 O H}$.

\begin{tabular}{|c|c|c|c|}
\hline & $10 H$ & $20 H$ & $30 H$ \\
\hline $\begin{array}{l}\text { Empirical } \\
\text { formula }\end{array}$ & $\mathrm{C}_{15} \mathrm{H}_{19} \mathrm{~N}_{3} \mathrm{O}_{2}$ & $\mathrm{C}_{16} \mathrm{H}_{21} \mathrm{~N}_{3} \mathrm{O}_{2}$ & $\mathrm{C}_{17} \mathrm{H}_{23} \mathrm{~N}_{3} \mathrm{O}_{3}$ \\
\hline $\begin{array}{l}\text { Formula } \\
\text { weight }\end{array}$ & 273.33 & 287.36 & 317.38 \\
\hline Temperature & $295(2) \mathrm{K}$ & $295(2) \mathrm{K}$ & $200(2) \mathrm{K}$ \\
\hline Wavelength & $1.54178 \AA$ & $1.54178 \AA$ & $1.54178 \AA$ \\
\hline Crystal system & Monoclinic & Triclinic & Monoclinic \\
\hline Space group & $\mathrm{P} 2(1)$ & $\mathrm{P}-1$ & $\mathrm{P} 2{ }_{1} / \mathrm{n}$ \\
\hline \multirow{3}{*}{$\begin{array}{c}\text { Unit cell } \\
\text { dimensions }\end{array}$} & $\begin{array}{l}a=9.4598(2) \AA \\
\alpha=90^{\circ}\end{array}$ & $\begin{array}{l}a=7.4623(8) \AA \\
\alpha=100.622(9)^{\circ}\end{array}$ & $\begin{array}{l}a=9.3568(4) \AA \\
\alpha=90^{\circ}\end{array}$ \\
\hline & $\begin{array}{l}\mathrm{b}=7.61760(10) \AA \\
\beta=95.575(2)^{\circ}\end{array}$ & $\begin{array}{l}\mathrm{b}=7.9403(9) \AA \\
\beta=92.419(9)^{\mathrm{o}}\end{array}$ & $\begin{array}{l}\mathrm{b}=15.0678(6) \AA \\
\beta=100.597(4)^{\circ}\end{array}$ \\
\hline & $\begin{array}{l}c=10.0179(2) \AA \\
\gamma=90^{\circ}\end{array}$ & $\begin{array}{l}\mathrm{c}=14.3735(15) \AA \\
\gamma=112.362(10)^{\mathrm{o}}\end{array}$ & $\begin{array}{l}c=11.9128(5) \AA \\
\gamma=90^{\circ}\end{array}$ \\
\hline Volume, Z & $718.48(2) \AA^{3}, 2$ & $768.19(14) \AA^{3}, 2$ & $1650.90(12) \AA^{3}, 4$ \\
\hline $\begin{array}{c}\text { Density } \\
\text { (calculated) }\end{array}$ & $1.263 \mathrm{Mg} / \mathrm{m}^{3}$ & $1.242 \mathrm{Mg} / \mathrm{m}^{3}$ & $1.277 \mathrm{Mg} / \mathrm{m}^{3}$ \\
\hline $\begin{array}{l}\text { Absorption } \\
\text { coefficient }\end{array}$ & $0.693 \mathrm{~mm}^{-1}$ & $0.671 \mathrm{~mm}^{-1}$ & $0.721 \mathrm{~mm}^{-1}$ \\
\hline $\mathrm{F}\left(\begin{array}{lll}0 & 0 & 0\end{array}\right)$ & 292 & 308 & 680 \\
\hline $\begin{array}{c}\text { Crystal size } \\
\left(\mathrm{mm}^{3}\right)\end{array}$ & $0.25 \times 0.20 \times 0.15$ & $0.25 \times 0.20 \times 0.15$ & $0.20 \times 0.15 \times 0.10$ \\
\hline $\begin{array}{c}\Theta \text { range for } \\
\text { data collection }\end{array}$ & 4.43 to $67.97^{\circ}$ & 3.15 to $68.00^{\circ}$ & 4.78 to $67.99^{\circ}$ \\
\hline $\begin{array}{l}\text { Limiting } \\
\text { indices }\end{array}$ & $\begin{array}{l}-10 \leq \mathrm{h} \leq 11 \\
-9 \leq \mathrm{k} \leq 9 \\
-11 \leq 1 \leq 12\end{array}$ & $\begin{array}{l}-8 \leq \mathrm{h} \leq 8 \\
-8 \leq \mathrm{k} \leq 9 \\
-17 \leq 1 \leq 15\end{array}$ & $\begin{aligned}-11 & \leq \mathrm{h} \leq 10 \\
-18 & \leq \mathrm{k} \leq 17 \\
-14 & \leq 1 \leq 11\end{aligned}$ \\
\hline $\begin{array}{c}\text { Reflections } \\
\text { collection }\end{array}$ & 4668 & 5153 & 6078 \\
\hline $\begin{array}{l}\text { Independent } \\
\text { reflections }\end{array}$ & $2566[\mathrm{R}(\mathrm{int})=0.0143]$ & $2781[\mathrm{R}(\mathrm{int})=0.0352]$ & $3005[\mathrm{R}($ int $)=0.0225]$ \\
\hline $\begin{array}{l}\text { Completeness } \\
\text { to } \Theta=67.99^{\circ}\end{array}$ & $100.0 \%$ & $99.5 \%$ & $99.8 \%$ \\
\hline $\begin{array}{c}\text { Absorption } \\
\text { correction }\end{array}$ & $\begin{array}{l}\text { Semi-empirical from } \\
\text { equivalents }\end{array}$ & $\begin{array}{l}\text { Semi-empirical from } \\
\text { equivalents }\end{array}$ & $\begin{array}{l}\text { Semi-empirical from } \\
\text { equivalents }\end{array}$ \\
\hline
\end{tabular}




\begin{tabular}{|c|l|l|l|}
\hline $\begin{array}{c}\text { Max. and min. } \\
\text { transmission }\end{array}$ & 1.00000 and 0.75593 & 1.00000 and 0.58358 & 1.00000 and 0.90285 \\
\hline $\begin{array}{c}\text { Refinement } \\
\text { method }\end{array}$ & $\begin{array}{l}\text { Full-matrix } \\
\text { least-squares on } \mathrm{F}^{2}\end{array}$ & $\begin{array}{l}\text { Full-matrix } \\
\text { least-squares on } \mathrm{F}^{2}\end{array}$ & $\begin{array}{l}\text { Full-matrix } \\
\text { least-squares on } \mathrm{F}^{2}\end{array}$ \\
\hline $\begin{array}{c}\text { Data / restraints } \\
\text { / parameters }\end{array}$ & $2566 / 1 / 185$ & $2781 / 2 / 193$ & $3005 / 1 / 217$ \\
\hline $\begin{array}{c}\text { Goodness-of-fit } \\
\text { on } \mathrm{F}^{2}\end{array}$ & 0.786 & 1.168 & 1.014 \\
\hline $\begin{array}{c}\text { Final R indices } \\
{[\mathrm{I}>2 \sigma(\mathrm{I})]}\end{array}$ & $\mathrm{R} 1=0.0365, \mathrm{wR} 2=$ & $\begin{array}{l}\mathrm{R} 1=0.0881, \mathrm{wR} 2= \\
0.2219\end{array}$ & $\begin{array}{l}\mathrm{R} 1=0.0595, \quad \mathrm{wR} 2= \\
0.1673\end{array}$ \\
\hline $\begin{array}{c}\mathrm{R} \text { indices } \\
\text { (all data) }\end{array}$ & $\begin{array}{l}\mathrm{R} 1=0.0373, \mathrm{wR} 2= \\
\mathrm{R} 1=0.1336, \quad \mathrm{wR} 2=\end{array}$ & $\begin{array}{l}\mathrm{R} 1=0.0723, \quad \mathrm{wR} 2= \\
0.1793\end{array}$ \\
\hline $\begin{array}{c}\text { Largest diff. } \\
\text { peak and hole }\end{array}$ & 0.183 and $-0.161 \mathrm{e} . \AA^{-3}$ & 0.542 and $-0.313 \mathrm{e} . \AA^{-3}$ & 0.772 and $-0.262 \mathrm{e} . \AA^{-3}$ \\
\hline
\end{tabular}




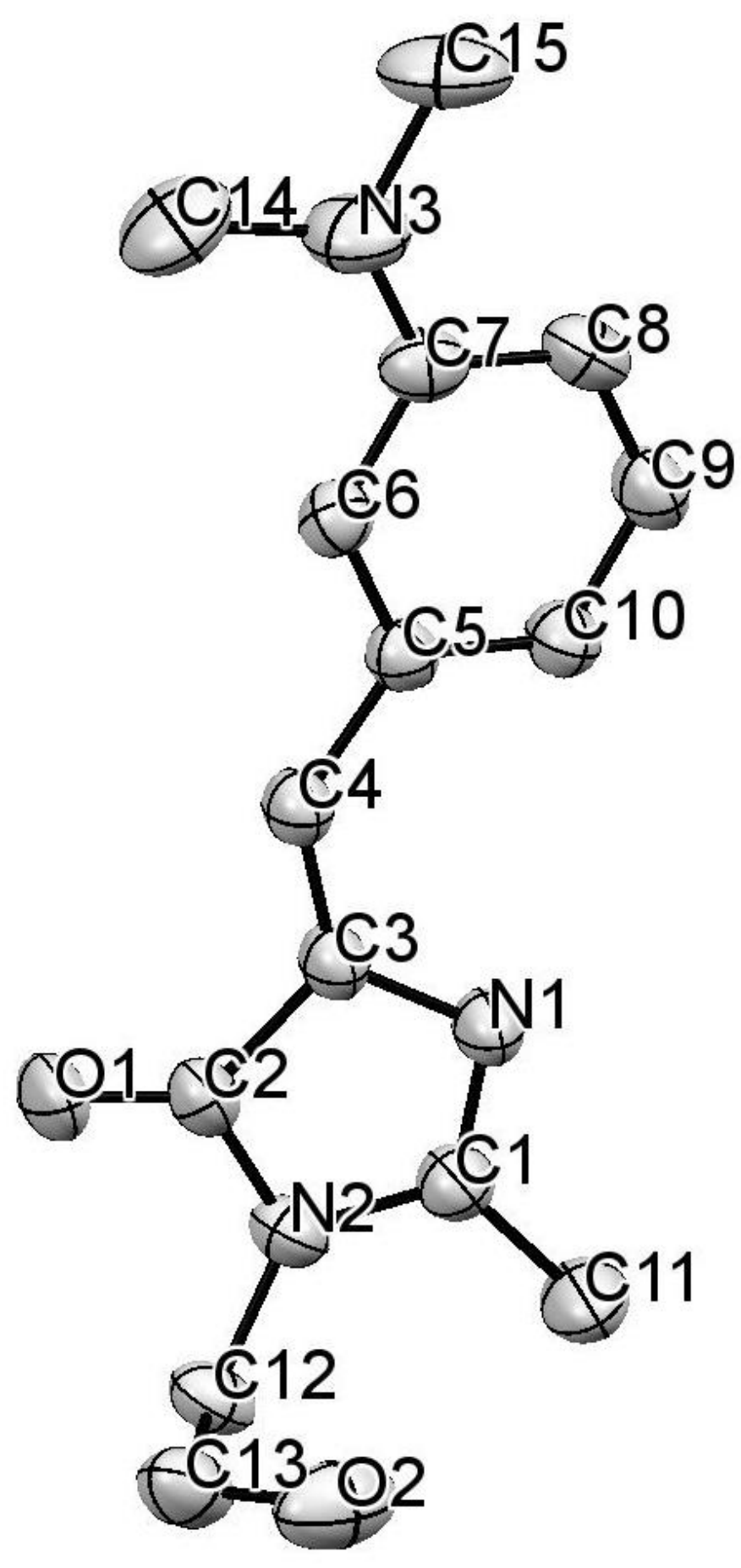

Figure S4. Thermal ellipsoid plot (30\%) of the crystal of $\mathbf{1 0 H}$. Hydrogen atoms are omitted for clarity. 


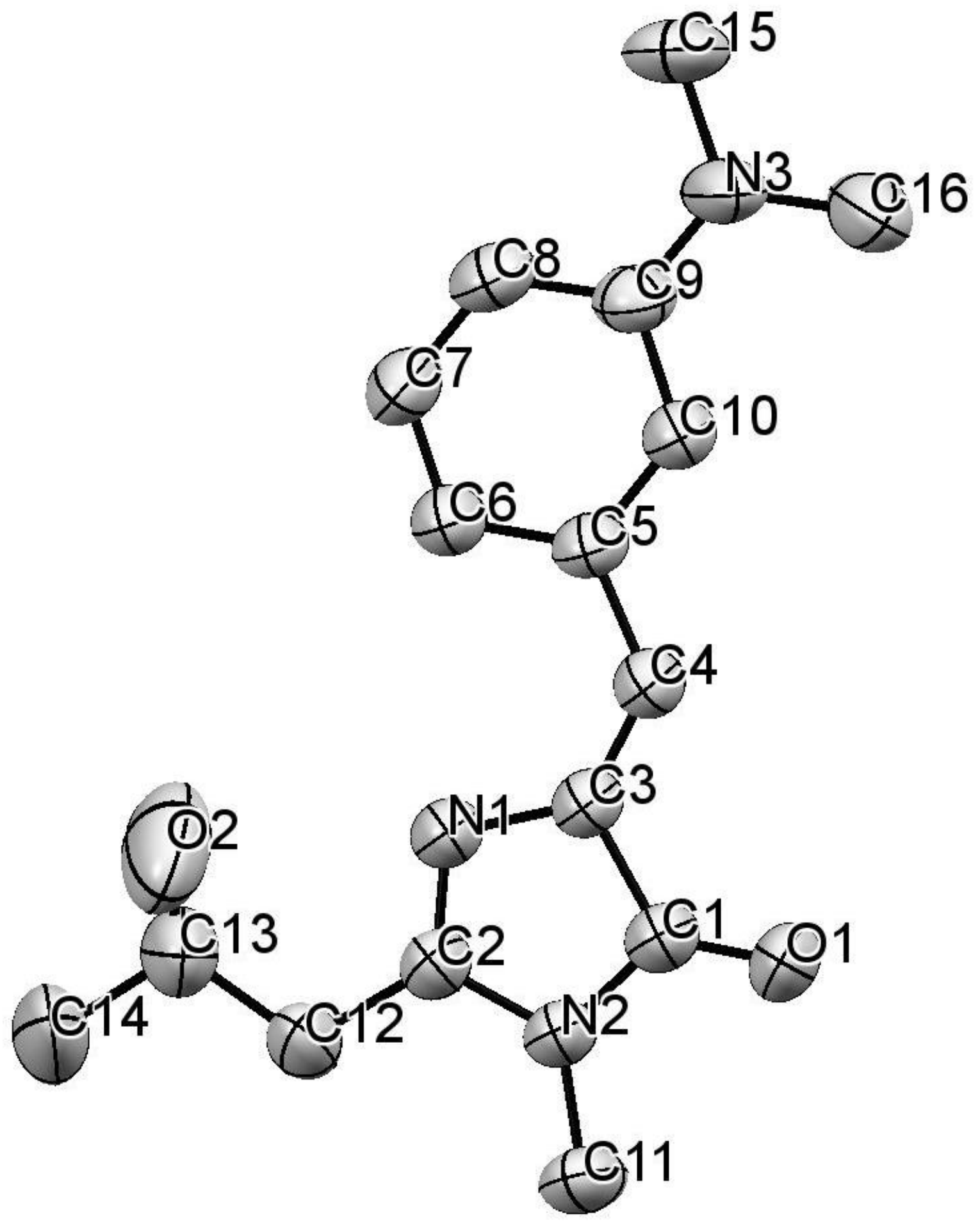

Figure S5. Thermal ellipsoid plot (30\%) of the crystal of $\mathbf{2 0 H}$. Hydrogen atoms are omitted for clarity. 


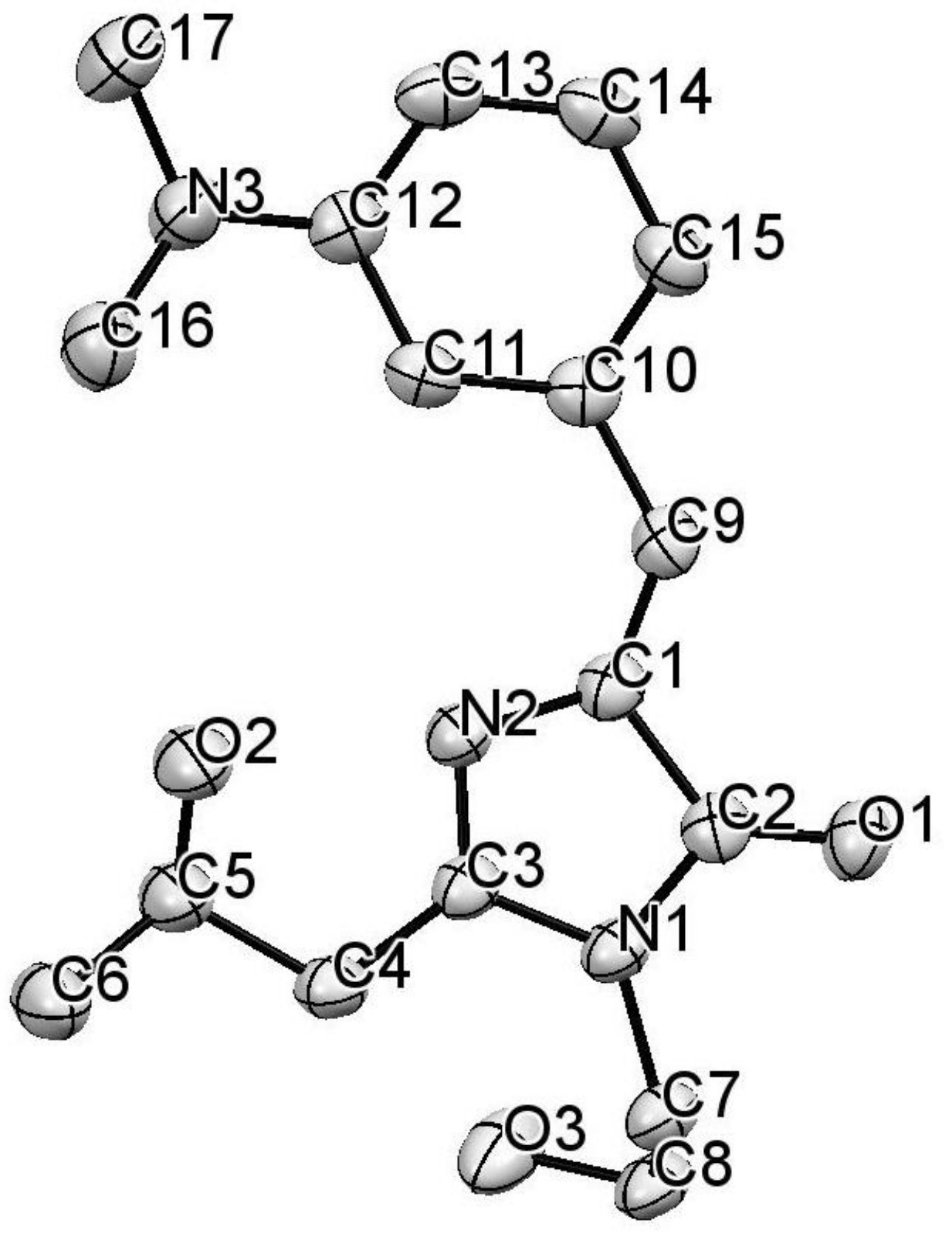

Figure S6. Thermal ellipsoid plot (30\%) of the crystal of $\mathbf{3 O H}$. Hydrogen atoms are omitted for clarity. 
Figure S7. Unit cell packing of $\mathbf{1 0 H}$.

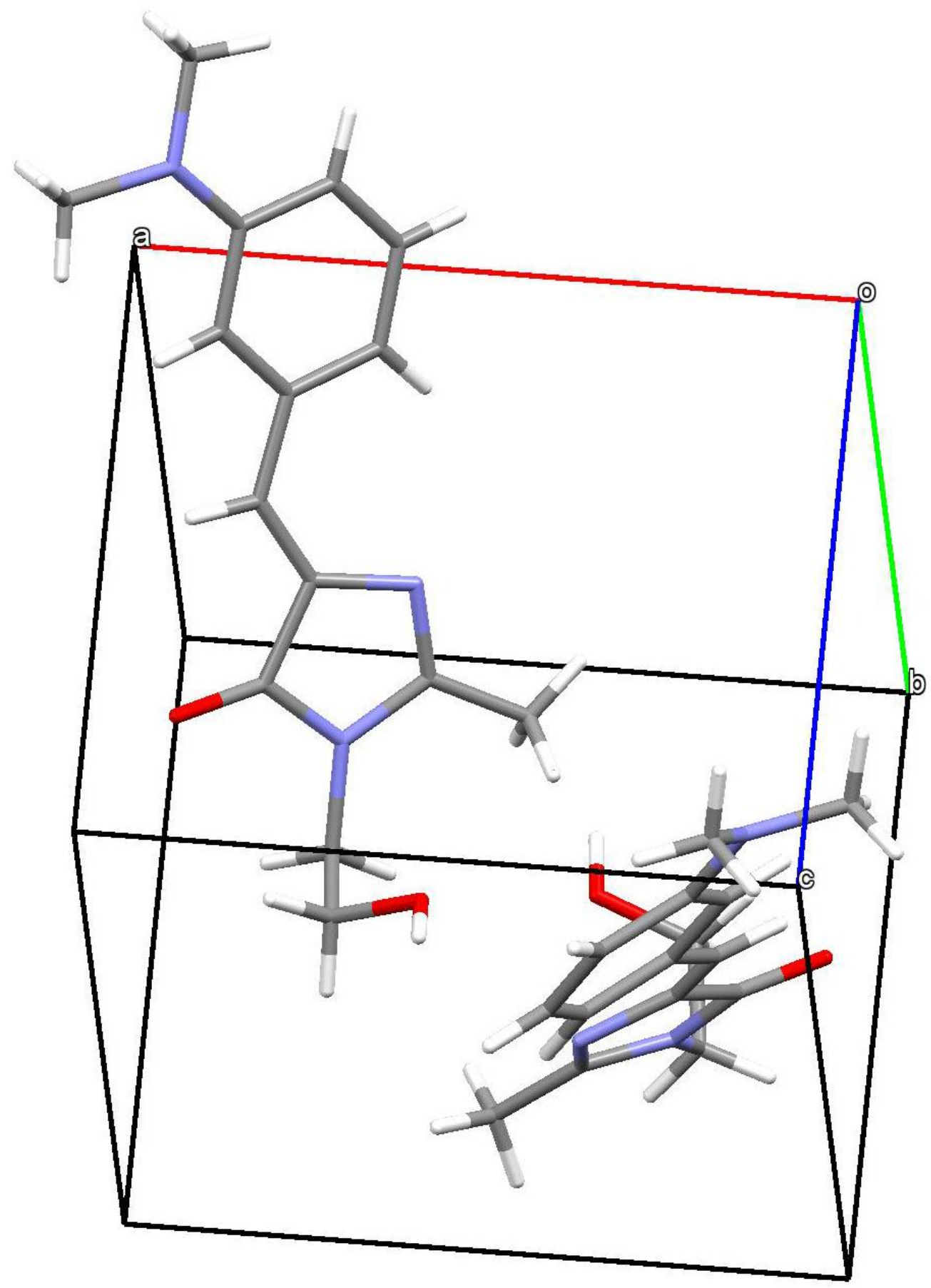


Figure S8. Unit cell packing of $\mathbf{2 O H}$.

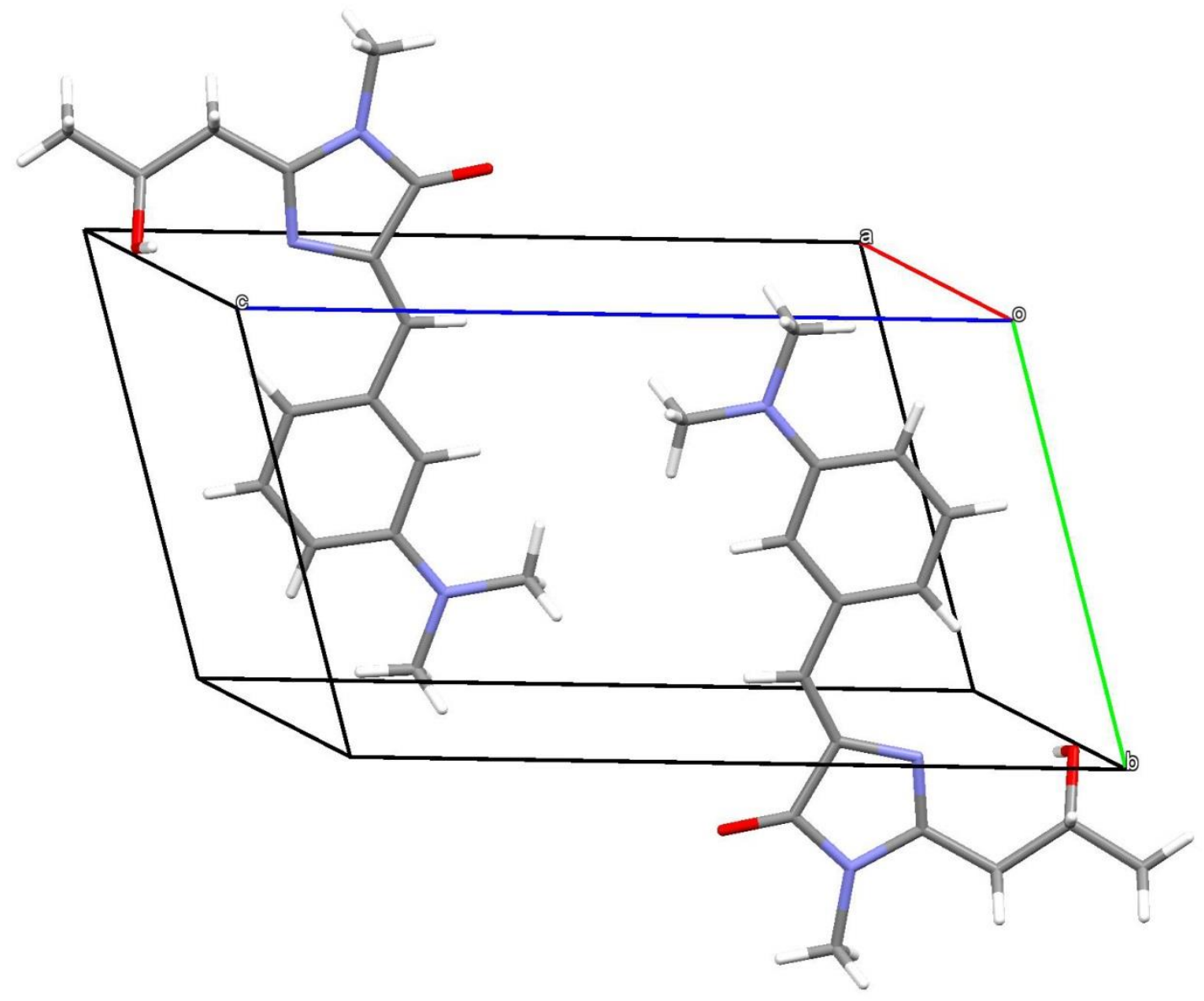


Figure S9. Unit cell packing of $\mathbf{3 O H}$.

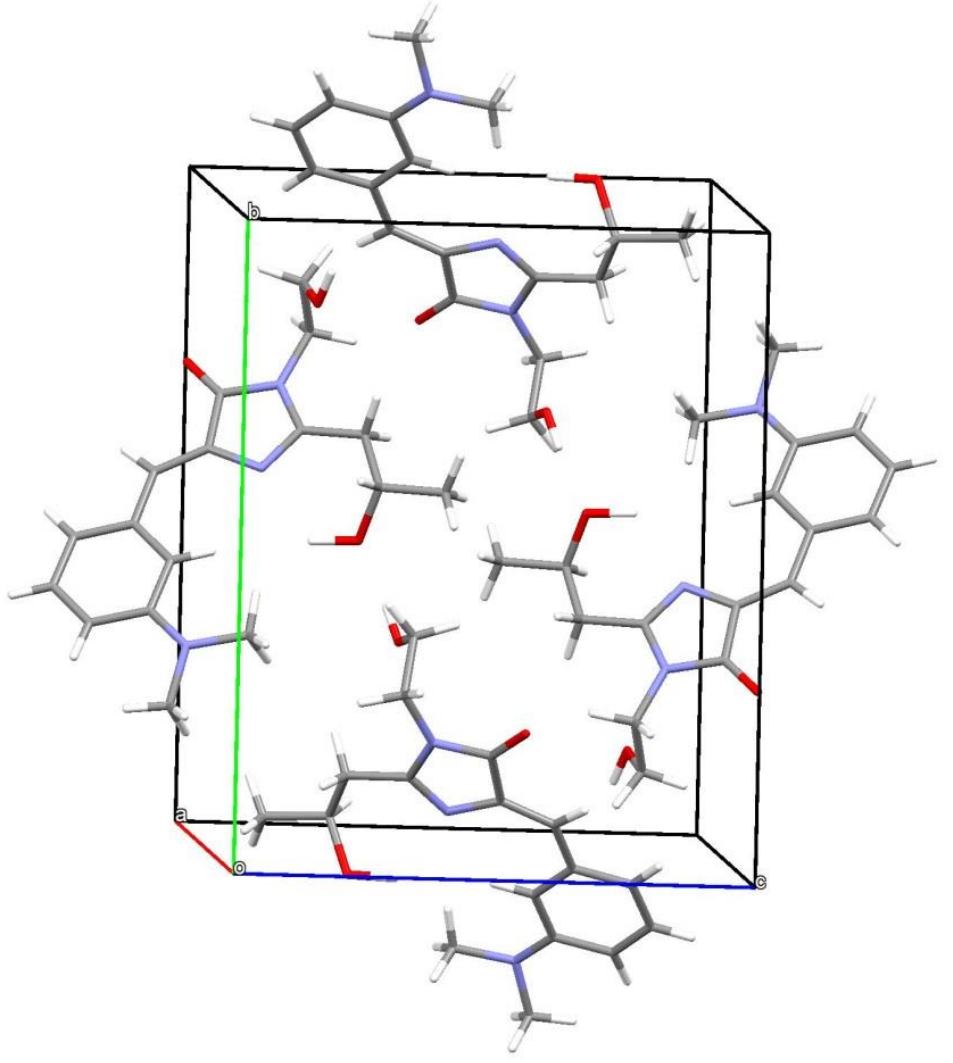


Figure S10. ${ }^{1} \mathrm{H} \mathrm{NMR}$ spectra of 4 in $\mathrm{CDCl}_{3}$.

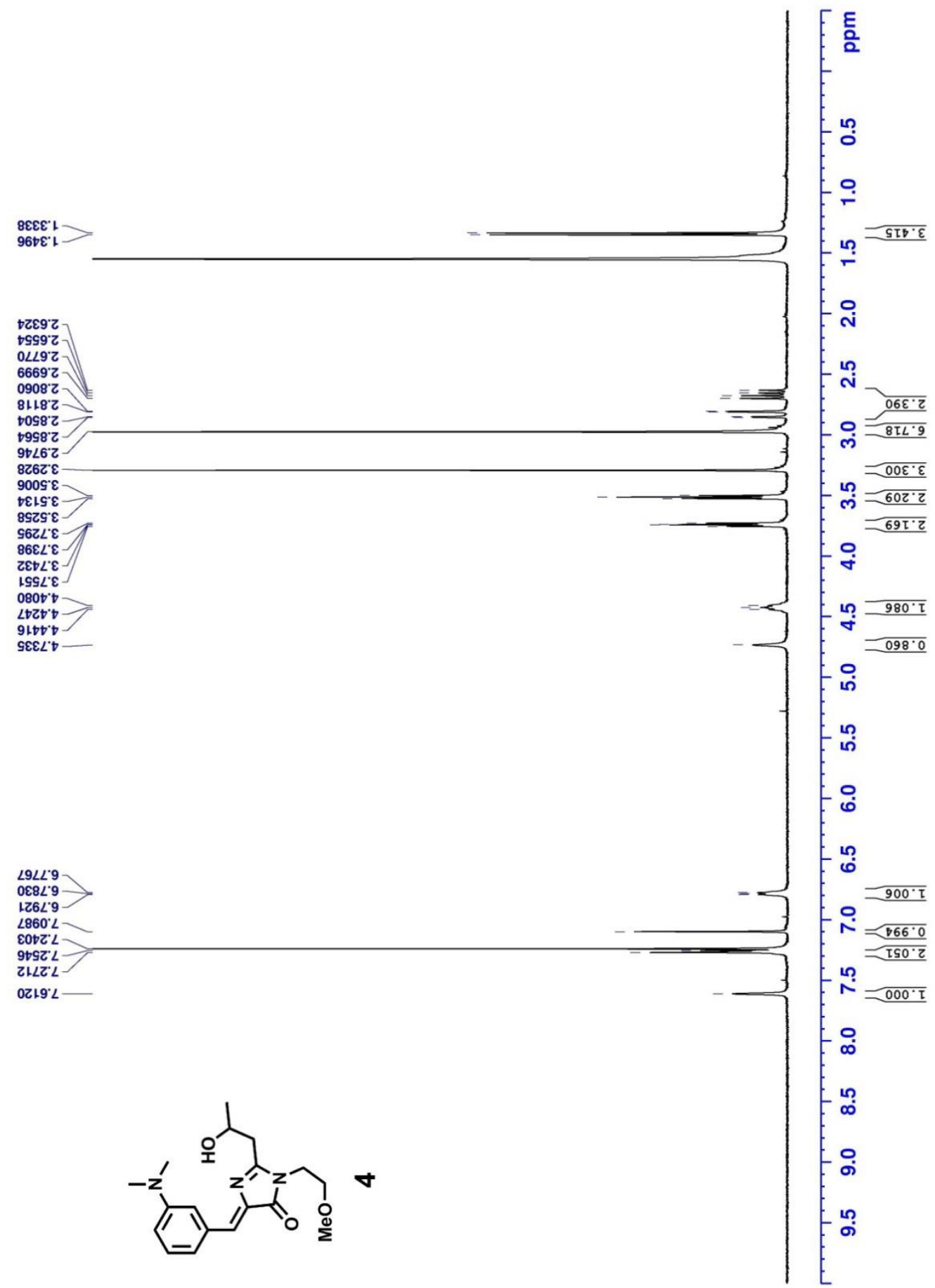


Figure S11. ${ }^{13} \mathrm{C}$ NMR spectra of 4 in $\mathrm{CDCl}_{3}$.

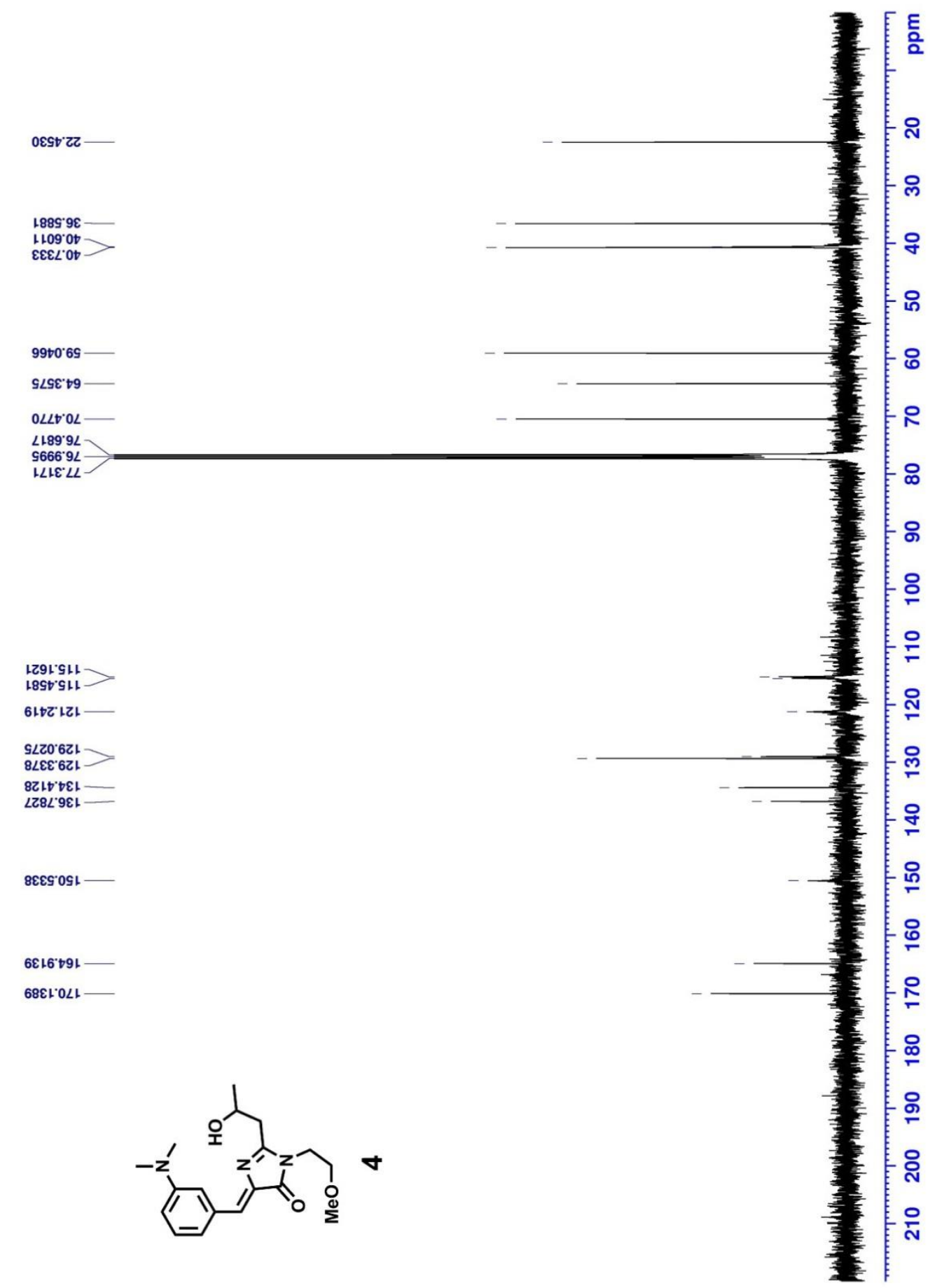


Figure S12. ${ }^{1} \mathrm{H}$ NMR spectra of $\mathbf{3 O M e}$ in $\mathrm{CDCl}_{3}$.

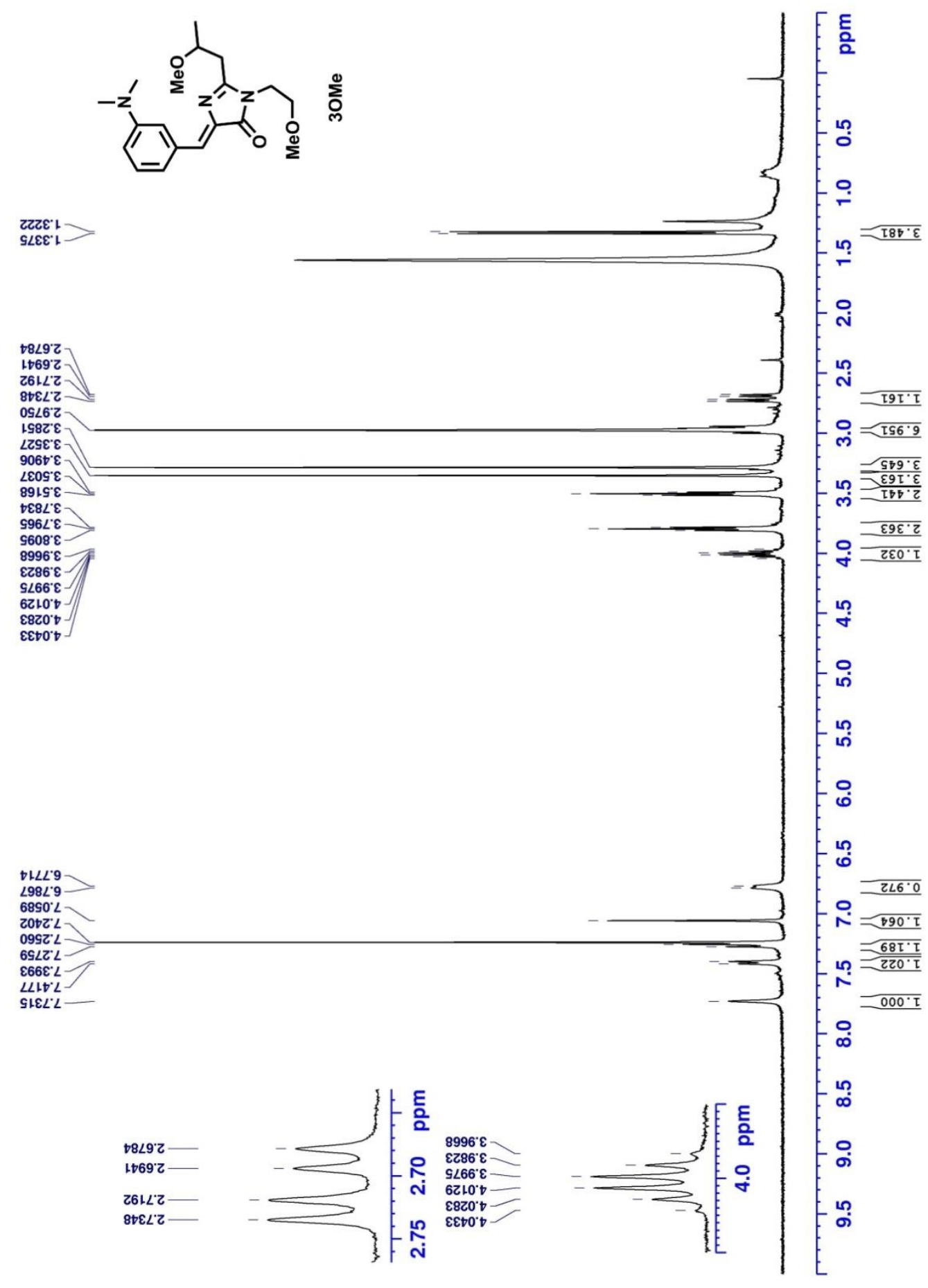


Figure S13. ${ }^{13} \mathrm{C}$ NMR spectra of 3OMe in $\mathrm{CDCl}_{3}$.

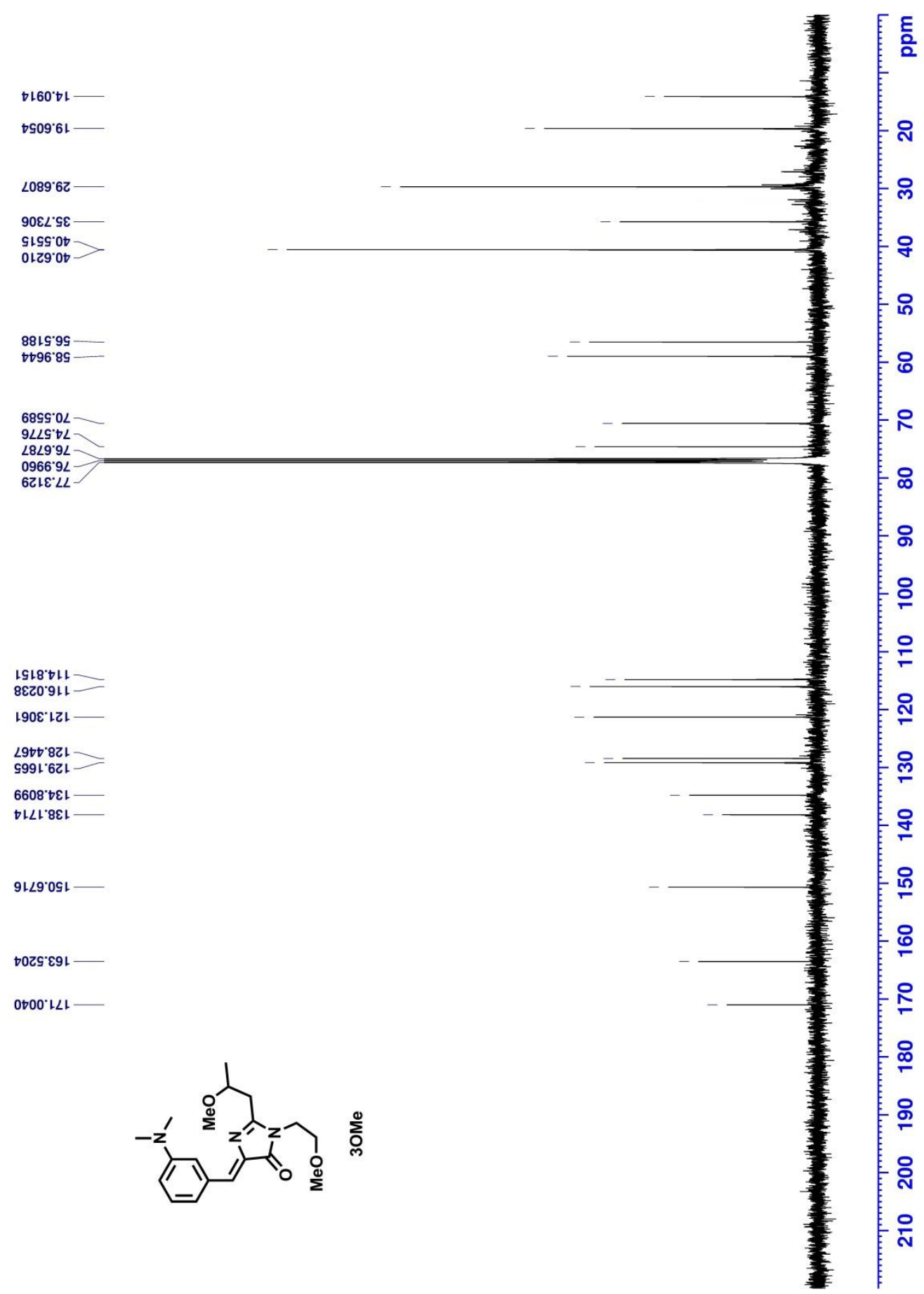


Figure S7. ${ }^{1} \mathrm{H}$ NMR spectra of $\mathbf{3 O H}$ in DMSO- $d_{6}$.

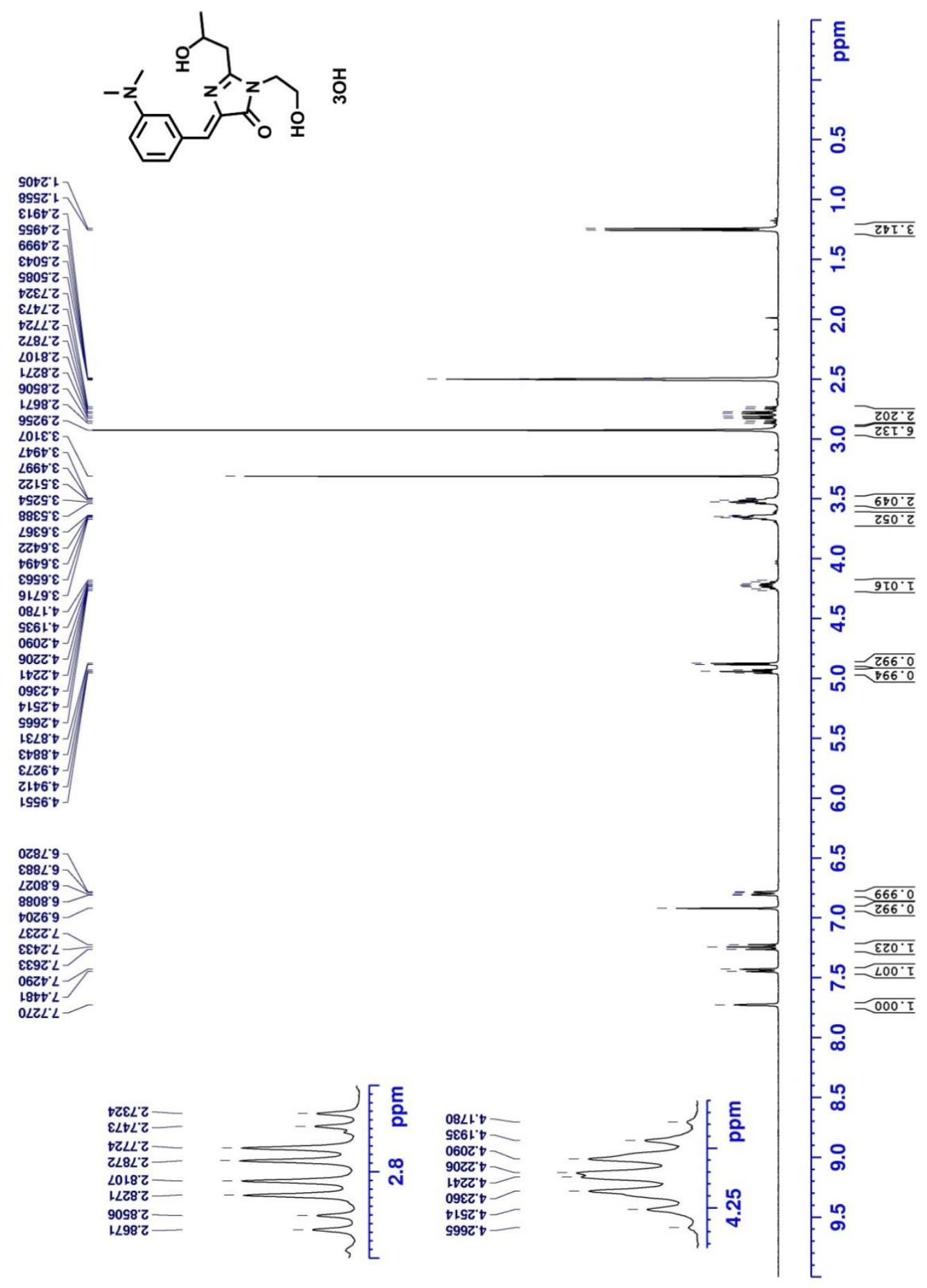


Figure S8. ${ }^{13} \mathrm{C}$ NMR spectra of $\mathbf{3 O H}$ in DMSO- $d_{6}$.

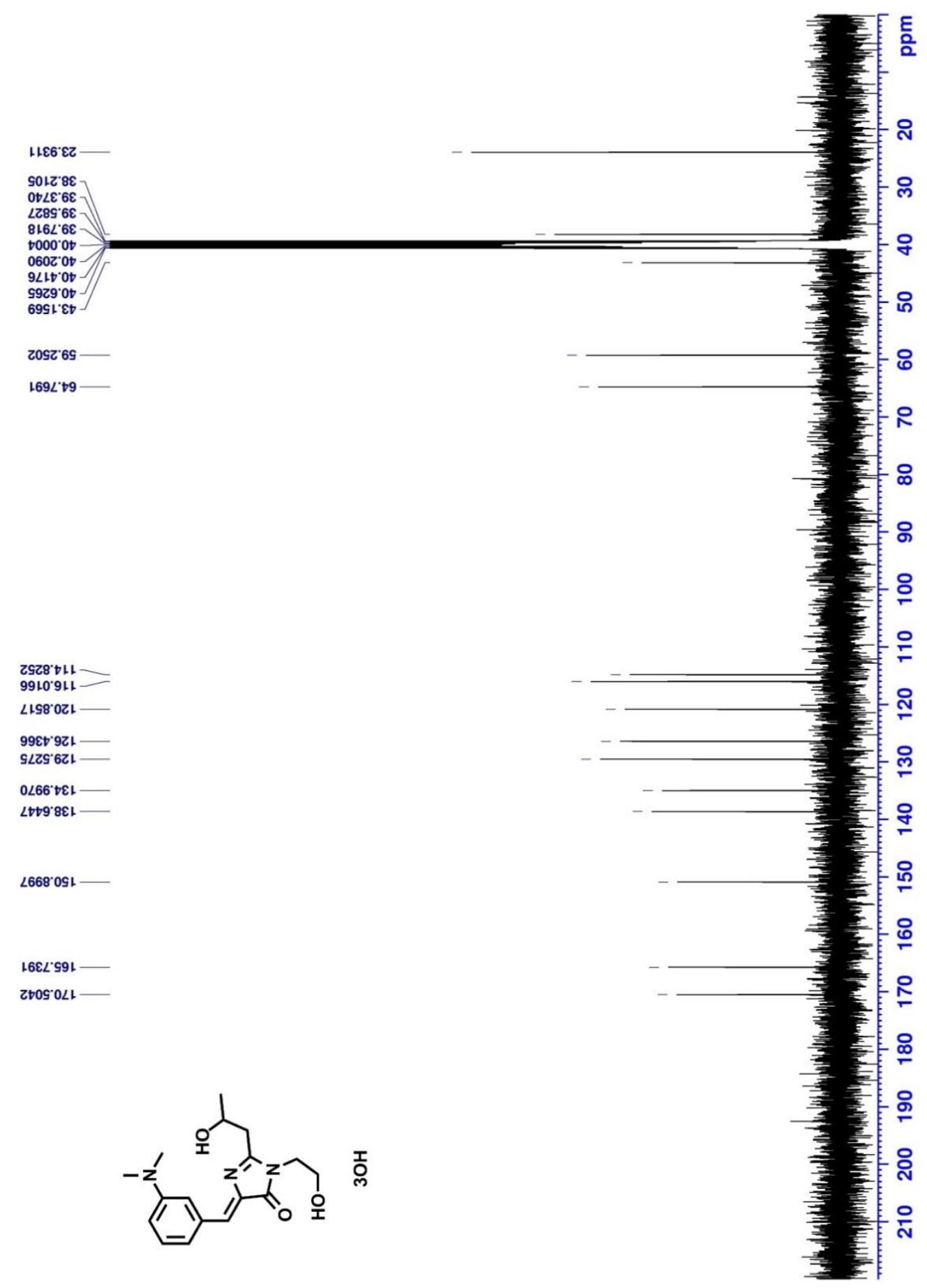

